\title{
Objective Comparison of Contour Detection in Noisy Images
}

\author{
Rodrigo Pavez, Marco Mora, and Paulo Gonzalez \\ Les Fous du Pixel \\ Image Processing Research Group \\ Department of Computer Science \\ Catholic University of Maule \\ Talca-Chile \\ rpavez@lfdp.org \{mora,pgonzalez\}@spock.ucm.cl \\ http://www.ganimides.ucm.cl/mmora
}

\begin{abstract}
The constant appearance of new contour detection methods makes it necessary to have accurate ways of assessing the performance of these methods. This paper proposes an evaluation method of contour detectors for noisy images. The method considers the computation of the optimal threshold that produces a greater approximation to the ground truth and the effect produced by the noise. Both analyzed dimensions allow objective comparisons of the performance of contour detectors.
\end{abstract}

\section{Introduction}

The contour detection is one of the most important problems in image processing. New operators and methods are constantly proposed with the aim of detecting contours in complex situations, for example: low contrast images, images in which objects have not precise and incomplete contours, images with high presence of noise, among others. The existence of different approaches to address the contour detection generates the need of evaluating such methods objectively and the need of comparing the performance of them.

To know the results of a contour detection method, a synthetic image from which the contours are known is considered. A human expert manually traces the contours, establishing what is known as a ground truth. This image is used as a benchmark to assess the outcome of a contour detection method.

To carry out the evaluation of a contour detector a performance function (PF) is required. This function is generally an expression that involves the amount of hits and errors on the ground truth. The performance function is usually normalized (values within a range), so it can be compared with the results of different methods.

When applying a contour detector, a dark image where the contours correspond to the lighter pixels is got. To assess the PF, it is necessary to threshold the image resulting from the application of the detector. Thus, we obtain a binary image, where the detected contour pixels differ completely from the background of the image. The number of hits and errors regarding the ground truth depends 
on the threshold value. Thus, the validity of the comparison depends on the correct computation of the threshold. The works from literature have varying degrees of depth to the computation of the threshold. There are proposals which compare results from different detectors with a determined threshold [1 2], where the validity of results is highly opened to criticism because they can change dramatically by choosing different thresholds. Other works compute the PF with a small set of thresholds 3456 , which does not permit to figure out if it is in the set where the threshold that produces the grater approximation to the ground truth is found. There are other works like [7], which considering the dependence of the PF on the threshold, perform a search process of the optimal threshold, thus allowing a more objective comparison.

The focus of this paper is the comparison of contour detection in noisy images. The approximation of previous works which were mentioned is enriched when studying the influence of noise in the computation of the appropriate threshold. A procedure to determine the optimal threshold considering different noise situations is proposed. The method enables to objectively compare the performance of contour detectors in images with high noise content.

The rest of the paper is organized as follows: Section 2 presents a new contour detector derived from the coefficient of variation $(\mathrm{CV})$, and the reasons why it is speculated that the new operator detects the contours better than the $\mathrm{CV}$ are outlined. Section 3 presents the objective method for evaluating contour detection. The procedure is applied to the detectors discussed in the preceding section, being clear the error in the conclusions. The final section presents the conclusions of the work.

\section{A New Contour Detector for Images with Multiplicative Noise}

The Coefficient of Variation is a value proposed as a contour detector in images with multiplicative noise 891011 . The $\mathrm{CV}$ is the ratio between the square root of standard deviation and the mean of a set of data, as shown in the following expression:

$$
C V=\frac{\sqrt{\sigma(W)}}{\bar{X}(W)}
$$

where $\mathrm{W}$ corresponds to a sample of data coming from the image. If the image has a high noise content, the analyzed window will define a population with a significant presence of outliers. A well-known fact is that the mean is not a good predictor of central tendencies when outliers are present. This means that the $\mathrm{CV}$ will not be a good contour detector if the images have a high noise content.

To address the above problem, the median can be used in populations with outliers. Following the above observation, the numerator and the denominator of the CV are modified based on the median in the following way: as a measure of deviation the Median Absolute Deviation (MAD) will be adopted, and as measure of central tendency the same median will be occupied. The new operator 
is called Modified Coefficient of Variation (MCV), and according to the previous reasoning, it should be robust than the $\mathrm{CV}$ for detecting contours in images with high content of multiplicative noise. The expressions of MAD and the MCV are presented in (2) and (3), respectively:

$$
\begin{gathered}
M A D(X)=\text { median }(|(X-\operatorname{median}(X))|) \\
M C V=\frac{M A D(W)}{\text { median }(W)}
\end{gathered}
$$

To generate an image that is contaminated with multiplicative noise a model widely accepted in literature will be used [12]. The expression of this model is:

$$
I_{n}=I_{w n} \times n
$$

where $I_{n}$ corresponds to the noisy image, $I_{w n}$ is the image without noise, and $n$ is the noise. It is noted that $n$ corresponds to a set of random numbers that follow a particular probability distribution. In this case, a Gaussian distribution of mean 1 and a standard deviation $\sigma$ is used. The value of $\sigma$ should be very little not to alter the original image significantly.

To compare the results of both detectors on the noisy image, a performance function that considers the hits and errors with respect to the ground truth is adopted 3 . The expression of the performance function is as follows:

$$
\rho=\frac{\operatorname{card}(P T)}{\operatorname{card}(P T)+\operatorname{card}(F P)+\operatorname{card}(F N)}
$$

where $\rho$ is the function of performance, $\operatorname{card}(X)$ is the cardinal of a set $\mathrm{X}, P T$ is the set of true positives, $F P$ is the set of false positives, and $F N$ is the set of false negatives. The performance function $\rho$ is close to 1 if there are many hits and few errors, and it is close to 0 if there are few hits and many errors.

An interesting approach for contour detection is to consider the contours as outliers of the image resulting from the application of a detector [13. This implies that contour detection is reduced to establish the values that are distant from the central tendency of the image. In this work, a simple but effective criterion has been adopted in order to determine the threshold, which separates the contour pixels from the rest of the image. The criterion is based on the median of the data as shown in the following expression:

$$
\text { th }=k \times \operatorname{median}\left(I_{c}\right)
$$

where $t h$ corresponds to the computed threshold, $k$ a constant that indicates the distance of the outlier in relation to a measure of central tendency, and $I_{c}$ the image resulting from the application of a contour detector.

Figure 1 shows the results of contour detection for the CV and the MCV. A synthetic image has been contaminated with multiplicative noise that follows a gaussian distribution with $\mu=0$ and $\sigma=0.125$. The threshold has been computed considering $k=2.5$ for the expression (6). From figures 1(e) and 1)(h), it can be 


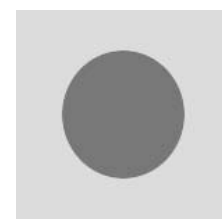

(a)

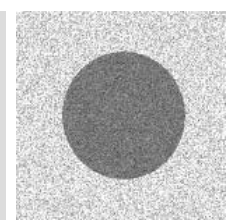

(b)

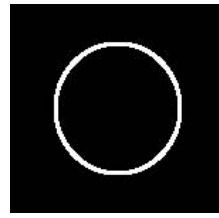

(c)

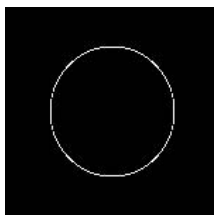

(f)

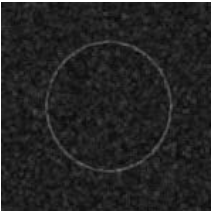

(d)

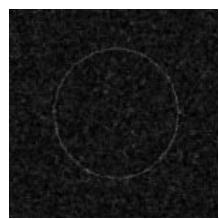

(g)

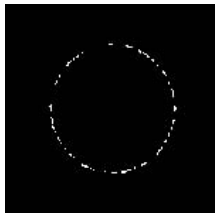

(e)

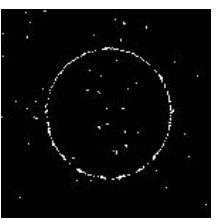

(h)

Fig. 1. Results of Contour Detection. Top row: (a) Original image (b) Noisy image with Gaussian distribution of the Noise. Middle row: (c) Original image contour with CV (d) Noisy image contour detection with CV (e) CV detected contours by thresholding. Down row: (f) Original image contour with MCV (g) Noisy image contour detection with MCV (h) MCV detected contours by thresholding.

visually seen that the MCV has a better performance than the CV. Numerically, the result is consistent since $\rho_{C V}<\rho_{M C V}\left(\rho_{C V}=0.1393, \mathrm{y} \rho_{M C V}=0.3618\right)$.

Figure 2 shows performance curves of the function $(\rho)$ for the $\mathrm{CV}$ and MCV, when varying the noise intensity. It starts from an image without noise $(\sigma=0)$, until an image with high level of noise is reached $(\sigma=0.2)$. The threshold that determines

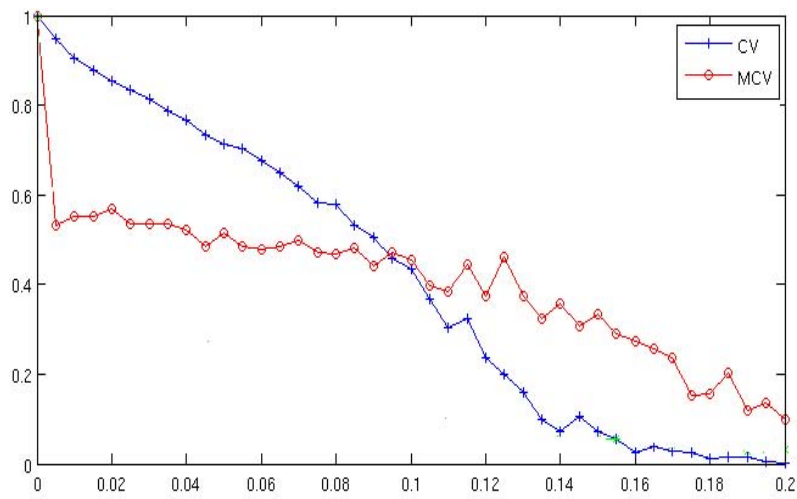

Fig. 2. Performance Function $(\rho)$ versus Noise Deviation $(\sigma)$ 
the detected contours is computed as $t h=2.5 \times$ median $\left(I_{c}\right)$. It is noted that as the noise grows, the PF of both detectors decreases. In the area of most intense noise, the $\mathrm{PF}$ of the MCV is higher than the PF of the CV, so it can be concluded that the MCV performs better than the CV on highly noisy images.

\section{Objective Comparison of Contour Detection in Noisy Images}

In the previous section, the benefits of the MCV as contour detector in images with multiplicative noise were shown. It was determined that for images with high noise level, the MCV performs better than the CV. Unfortunately, the conclusion has been obtained by performing a partial analysis of the variables that influence the performance of the detector.

In this paper, the effect that the threshold value has on the performance of the detector is analyzed as well as the fact of subjecting the image to different

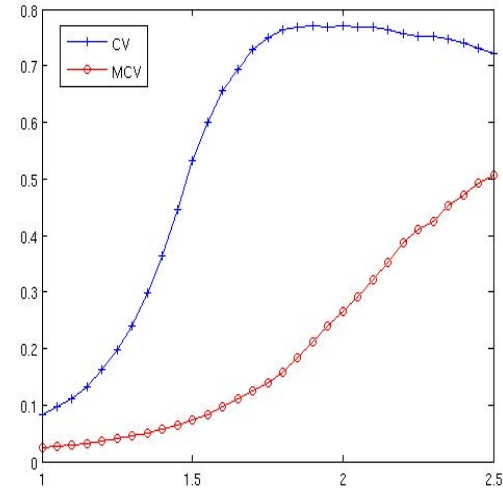

(a) $\sigma=0.05$

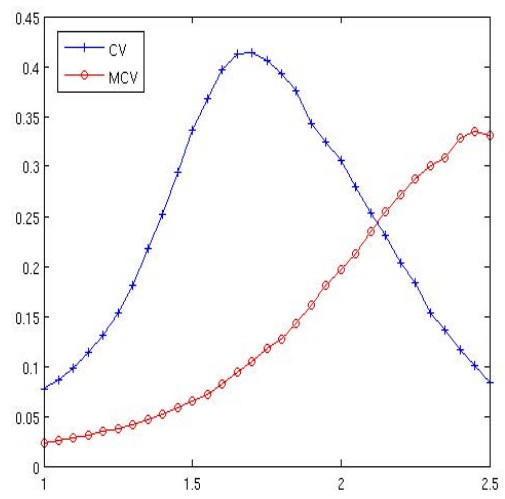

(c) $\sigma=0.15$

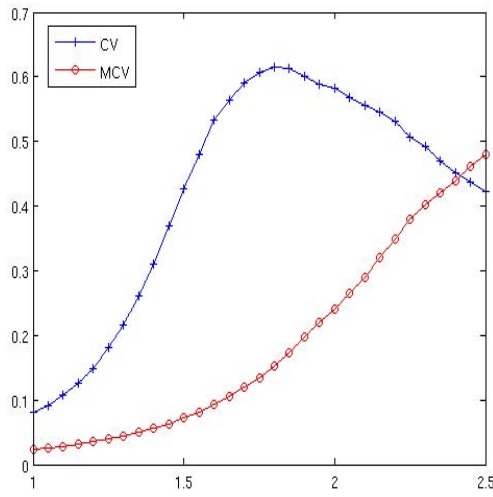

(b) $\sigma=0.1$

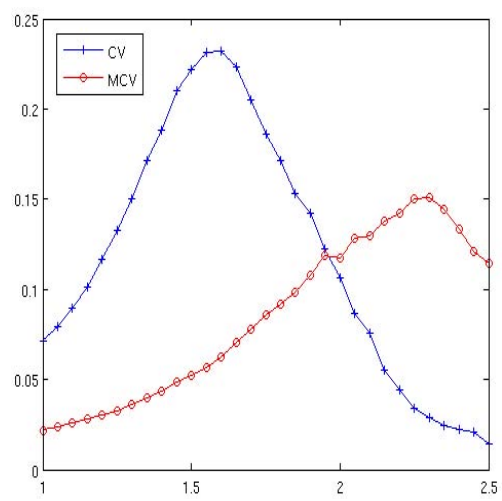

(d) $\sigma=0.2$

Fig. 3. Behavior of performance function with variable threshold $(1<k<2.5)$ in 4 noise intensity cases 
intensities of noise. The objective analysis of the performance of a contour detector consists of finding the threshold that maximizes the performance function (optimal threshold) for different noise situations.

Figure 3 shows the value of the performance function of the $\mathrm{CV}$ and $\mathrm{MCV}$ for a variable threshold variable in 4 different noise situations. The threshold is determined by the constant $k$, and the noise is defined by the standard deviation $\sigma$ of the noise distribution. It is clearly observed that, in case of noise, the higher value of the performance function always corresponds to the CV. This figure shows that for these 4 cases of noise, the $\mathrm{CV}$ detects the contours better than the MCV, information which contrasts with the results of the previous section.

In order to generalize the previous result, figure 4 presents the curve of the higher value of performance function for a continuous flow of noise case, both for the $\mathrm{CV}$ and $\mathrm{MCV}$. From the figure, it can be seen that the value of the performance function associated to the optimal threshold is always higher for the $\mathrm{CV}$ (the curve for the $\mathrm{CV}$ is always above the curve of the MCV). This figure is an objective test to evaluate and compare the performance of the detectors in question. This allows us to conclude that the $\mathrm{CV}$ is a contour detector stronger than the MCV for images with multiplicative noise.

Figure 5 shows the contours detected for 3 levels of noise with $\mathrm{CV}$ and MCV. The top row corresponds to a low noise level $(\sigma=0.1)$, the central line to a medium noise level $(\sigma=0.15)$, the bottom row to a high noise level $(\sigma=0.2)$. The columns of the figure show the noisy images, the contours detected by the CV and MCV, respectively. In the caption of noisy images, the noise standard deviation is $\sigma$. In the caption of the detected contour images, the optimal threshold is indicated by the constant $k$, and the maximum value of the performance function by $\rho$. First, the figure shows that when the noise increases, the errors in the detected contours increase

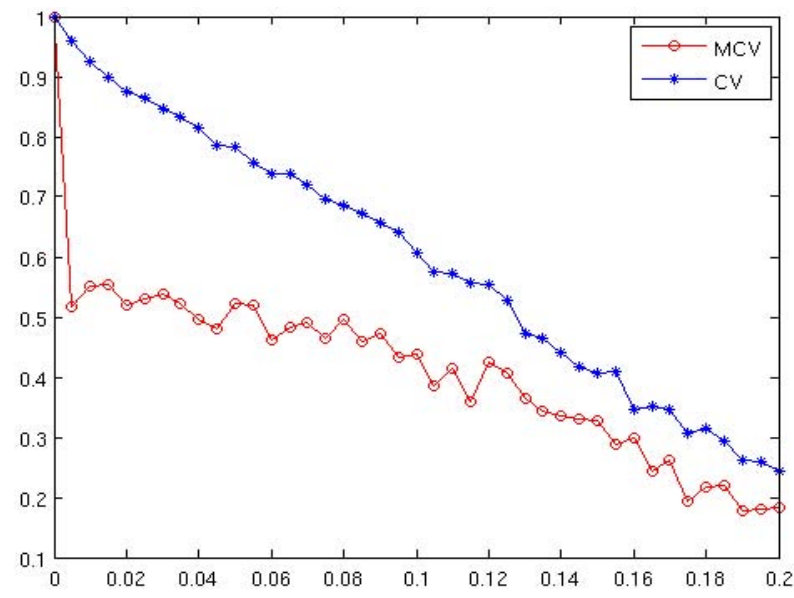

Fig. 4. Maximum values of the performance function computed with variable noise $(0<\sigma<0.2)$ 
as well, what explains the decline of the curve with maximum value of the performance function. Secondly, when observing the numerical indicators, it is confirmed that the CV has a higher value for the performance function $\rho$ than the MCV, no matter the level of noise of the image. Finally, the figure shows that by visual inspection, it is very difficult to decide which method has closer results on the ground truth. This underscores the importance of an objective and numerical comparison of the results of contour detection.

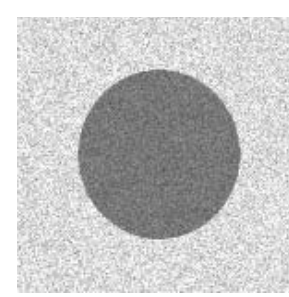

(a) $\sigma=0.1$

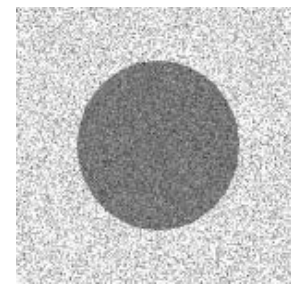

(d) $\sigma=0.15$

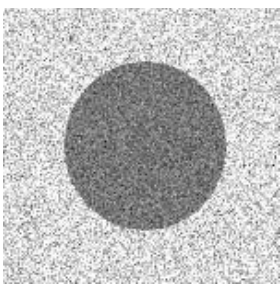

(g) $\sigma=0.2$

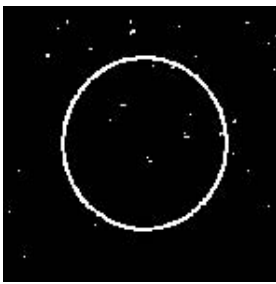

(b) $\mathrm{k}=1.8, \rho=0.60$

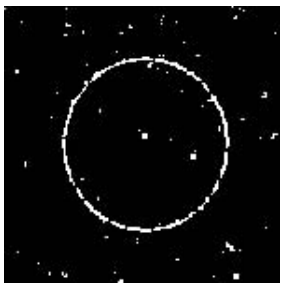

(e) $\mathrm{k}=1.7, \rho=0.40$

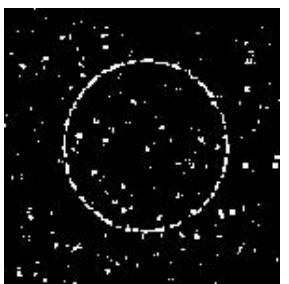

(h) $\mathrm{k}=1.6, \rho=0.24$

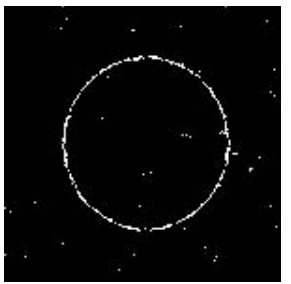

(c) $\mathrm{k}=2.5, \rho=0.44$

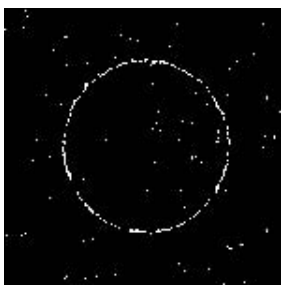

(f) $\mathrm{k}=2.4, \rho=0.33$

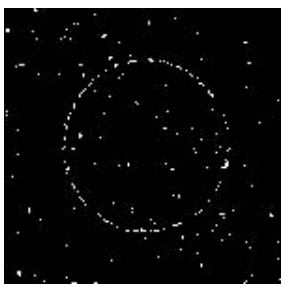

(i) $\mathrm{k}=2.4, \rho=0.18$

Fig. 5. Results of $\mathrm{CV}$ and MCV detected contours with three levels of noise. Top row: Low level of noise (a) Noisy image (b) CV detected contours (c) MCV detected contours. Middle row: Medium level of noise (d) Noisy image (e) CV detected contours (f) MCV detected contours. Down row: High level of noise (g) Noisy image (h) CV detected contours (i) MCV detected contours.

\section{Conclusions}

A method to compare the result of contour detection in noisy images in an objective way has been presented in this article. The method considers the influence of the threshold that determines the contours which have been detected, and the 
noise level of the image. As an example, the comparison between two detectors (CV and MCV) for images with multiplicative noise has been developed.

Initially, the CV and MCV were compared by a method with partial information, which led to erroneous conclusions. It is shown that with the proposed method an objective comparison can be made, what permits to draw accurate conclusions when evaluating the performance of contour detectors.

\section{References}

1. Pagador, J., Moreno, J., Masero, V., León-Rojas, J.: Active Contour on the Basis of Inertia. In: Proceedings of the 2004 ACM Symposium on Applied Computing, pp. 307-308 (2004)

2. Yu, L., Ma, F., Jayasuriya, A., Sigelle, M., Perreau, S.: A New Contour Detection Approach in Mammogram using Rational Wavelet Filtering and MRF Smoothing. In: Proceedings of the 9th Biennial Conference of the Australian Pattern Recognition Society on Digital Image Computing Techniques and Applications, pp. 106-111 (2007)

3. Grigorescu, C., Petkov, N., Westenberg, M.: Contour Detection based on Nonclassical Receptive Field Inhibition. IEEE Transactions on Image Processing 12, 729-739 (2003)

4. Joshi, G., Sivaswamy, J.: A Simple Scheme for Contour Detection. In: International Conference on Computer Vision Theory and Applications (VISAPP 2006), pp. 236-242 (2006)

5. Farag, A., Suri, J., Ferrari, R., Rangayyan, R., Desautels, J., Frère, A., Borges, R.: Detection of the Breast Contour in Mammograms by Using Active Contour Models. In: Deformable Models. Topics in Biomedical Engineering International Book Series, pp. 133-162 (2007)

6. Chabrier, S., Laurent, H., Rosenberger, C., Emile, B.: Comparative Study of Contour Detection Evaluation Criteria based on Dissimilarity Measures. EURASIP Journal on Image and Video Processing (2008)

7. Wang, S., Ge, F., Liu, T.: Evaluating Edge Detection Through Boundary Detection. EURASIP Journal on Applied Signal Processing, 1-15 (2006)

8. Lee, J.: Digital Image Enhancement and Noise Filtering by Using Local Statistic. IEEE Transactions on Pattern Analysis and Machine Intelligence PAMI-2, 165-168 (1980)

9. Frost, V., Stiles, J., Shanmugan, K., Holtzman, J.: A Model for Radar Images and its Application to Adaptive Digital Filtering of Multiplicative Noise. IEEE Transactions on Pattern Analysis and Machine Intelligence PAMI-4, 157-166 (1982)

10. Kuan, D., Sawchuk, A., Strand, T., Chavel, P.: Adaptive Restoration of Images with Speckle. IEEE Transaction on Acoustics, Speech and Signal Processing 35, 373-383 (1987)

11. Lopes, A., Touzi, R., Nezry, E.: Adaptive Speckle Filters and Scene Heterogeneity. IEEE Transactions on Geoscience and Remote Sensing 28, 992-1000 (1990)

12. Krissian, K., Westin, C.F., Kikinis, R., Vosburgh, K.: Oriented Speckle Reducing Anisotropic Diffusion. IEEE Transaction on Image Processing 16, 1412-1424 (2007)

13. Black, M., Sapiro, G.: Edges as Outliers: Anisotropic Smoothing Using Local Image Statistics. In: Nielsen, M., Johansen, P., Fogh Olsen, O., Weickert, J. (eds.) ScaleSpace 1999. LNCS, vol. 1682, pp. 259-270. Springer, Heidelberg (1999) 